\title{
Astragaloside IV alleviates liver injury in type 2 diabetes due to promotion of AMPK/mTOR-mediated autophagy
}

\author{
YUNFENG ZHU $^{1 *}$, YONG SU $^{2 *}$, JIE ZHANG $^{1 *}$, YANHUA ZHANG $^{1}$, YAN LI $^{1}$, \\ YULI HAN ${ }^{1}$, XIANAN DONG ${ }^{1}$, WEIZU LI ${ }^{1}$ and WEIPING LI ${ }^{1,3}$ \\ ${ }^{1}$ Key Laboratory of Anti-Inflammatory and Immunopharmacology, Ministry of Education, \\ Department of Pharmacology, Basic Medicine College, Anhui Medical University; ${ }^{2}$ Department of Pharmacy, \\ The First Affiliated Hospital of Anhui Medical University, Hefei, Anhui 230032; ${ }^{3}$ Department of Pharmacology, \\ Anqing Medical and Pharmaceutical College, Anqing, Anhui 246052, P.R. China
}

Received November 16, 2020; Accepted March 16, 2021

DOI: $10.3892 / \mathrm{mmr} .2021 .12076$

\begin{abstract}
Diabetic liver injury is a serious complication of type 2 diabetes mellitus (T2DM), which is often irreversible in the later stage, and affects the quality of life. Autophagy serves an important role in the occurrence and development of diabetic liver injury. For example, it can improve insulin resistance (IR), dyslipidaemia, oxidative stress and inflammation. Astragaloside IV (AS-IV) is a natural saponin isolated from the plant Astragalus membranaceus, which has comprehensive pharmacological effects, such as anti-oxidation, anti-inflammation and anti-apoptosis properties, as well as can enhance immunity. However, whether AS-IV can alleviate diabetic liver injury in T2DM and its underlying mechanisms remain unknown. The present study used high-fat diets combined with low-dose streptozotocin to induce a diabetic
\end{abstract}

Correspondence to: Professor Weiping Li or Professor Weizu Li, Key Laboratory of Anti-Inflammatory and Immunopharmacology, Ministry of Education, Department of Pharmacology, Basic Medicine College, Anhui Medical University, 81 Meishan Road, Hefei, Anhui 230032, P.R. China

E-mail: 1wp19@126.com

E-mail: liweizu@126.com

*Contributed equally

Abbreviations: AS-IV, Astragaloside IV; T2DM, type 2 diabetes mellitus; HFD, high-fat diets; STZ, streptozotocin; IR, insulin resistance; AMPK, adenosine monophosphate-activated protein kinase; $\mathrm{CMC}-\mathrm{Na}^{+}$, carboxyl methyl cellulose sodium; FBG, fasting blood glucose; Met, Metformin; OGTT, oral glucose tolerance test; AUC, area under the curve; ALT, alanine aminotransferase; AST, aspartate aminotransferase; TC, total cholesterol; TG, triglyceride; HDL-C, high-density lipoprotein cholesterol; LDL-C, low-density lipoprotein cholesterol; GSP, glycosylated serum protein; FINS, fasting insulin; HOMA-IR, homeostasis model assessment of insulin resistance; HO-1, heme oxygenase-1

Key words: AS-IV, diabetic liver injury, autophagy, AMPK, mTOR liver injury model in T2DM rats to investigate whether AS-IV could alleviate diabetic liver injury and to identify its underlying mechanisms. The results demonstrated that AS-IV treatment could restore changes in food intake, water intake, urine volume and body weight, as well as improve liver function and glucose homeostasis in T2DM rats. Moreover, AS-IV treatment promoted suppressed autophagy in the liver of T2DM rats and improved IR, dyslipidaemia, oxidative stress and inflammation. In addition, AS-IV activated adenosine monophosphate-activated protein kinase (AMPK), which inhibited mTOR. Taken together, the present study suggested that AS-IV alleviated diabetic liver injury in T2DM rats, and its mechanism may be associated with the promotion of AMPK/mTOR-mediated autophagy, which further improved IR, dyslipidaemia, oxidative stress and inflammation. Thus, the regulation of autophagy may be an effective strategy to treat diabetic liver injury in T2DM.

\section{Introduction}

Type 2 diabetes mellitus (T2DM) is a severe and lifelong metabolic disease that is often characterised by insulin resistance (IR) and reduced insulin production, resulting in abnormally elevated blood glucose levels (1). It is estimated that by 2040 , the number of individuals suffering from diabetes worldwide will reach 642 million, of which T2DM will account for $\sim 90 \%$ (2). Previous studies have reported that metabolic disorders associated with T2DM could lead to liver injury, eventually resulting in a series of liver diseases such as fatty liver, cirrhosis and hepatocellular carcinoma (3-5). Diabetic liver injury is a major complication of T2DM caused by a variety of factors, such as hyperglycaemia, IR, dyslipidaemia, oxidative stress and inflammation (5). Among them, dyslipidaemia, oxidative stress and inflammation are conceived as the most common factors contributing to IR, which promotes the progression of T2DM (6). However, the exact molecular mechanism of diabetic liver injury in T2DM is not fully understood.

Autophagy is the major degradation and dynamic circulation system in the cell, via which cytoplasmic materials are transferred to, and degraded in the lysosome for cellular 
renovation and homeostasis (7). The impairment of autophagy with IR can be observed in obesity-induced diabetes, and the recovery of the autophagic flux can improve IR in T2DM (8). Moreover, autophagy not only regulates lipid metabolism but also reduces oxidative stress and inflammation (9-11). Thus, autophagy may be closely associated with the occurrence and development of diabetic liver injury in T2DM. Adenosine monophosphate-activated protein kinase (AMPK), a major cellular energy sensor, is a key factor in maintaining metabolic homeostasis (12). Accumulating evidence has revealed the close relationship between AMPK and autophagy, and AMPK can promote autophagy by inhibiting the mTOR pathway, which directly dephosphorylates and inhibits downstream 70-kD ribosomal protein S6 kinase (13-15). Therefore, AMPK/mTOR-mediated autophagy may be an ideal target for the treatment of diabetic liver injury in T2DM.

Astragaloside IV (AS-IV) is a natural saponin isolated from the plant Astragalus membranaceus. Recent studies have revealed that AS-IV has anti-oxidation, anti-inflammation and anti-apoptosis effects, and can enhance immunity (16-18). Our previous studies also showed that AS-IV exerts protective effects on the endoplasmic reticulum stress-induced apoptosis of renal tubular epithelial cells and diabetic cardiomyopathy in T2DM rats $(18,19)$. However, it remains unknown whether AS-IV exerts a protective effect on diabetic liver injury in T2DM, and whether autophagy is involved in diabetic liver injury is yet to be elucidated. Therefore, the present study was performed to investigate the protective effect and molecular mechanism of AS-IV on diabetic liver injury by regulating AMPK/mTOR-mediated autophagy in T2DM rats. Importantly, this study may provide a potential therapy using AS-IV in the prevention of diabetic liver injury in T2DM.

\section{Materials and methods}

Drug preparation. AS-IV (purity $>98 \%$; Nanjing Zelang Pharmaceutical Technology Co., Ltd.) and metformin hydrochloride (Met; Shanghai Shangyao Xinyi Pharmaceutical Co. Ltd.) were suspended in $0.5 \%$ carboxyl methyl cellulose sodium $\left(\mathrm{CMC}-\mathrm{Na}^{+}\right)$aqueous solution as an administration vehicle.

Animals and treatment. Male Sprague-Dawley rats (age, 6-8 weeks; weight, $200 \pm 20 \mathrm{~g}$; $\mathrm{n}=36$ ) were purchased from the Shandong Experimental Animal Center (Shandong, China) and housed in an environment at $24 \pm 2^{\circ} \mathrm{C}$ and $60 \pm 5 \%$ humidity with free access to water and standard diet under a 12-h light/dark cycle. All animal experiments were conducted under protocols approved by the Ethics Review Committee of Anhui Medical University (approval no. LLSC20190302).

The rats were acclimatised for 1 week before the experiment and randomly allocated to a normal control group $(n=6)$ and a diabetic group $(n=30)$. In the diabetic group, rats were fed with high-fat diets (HFD) containing 59.5\% standard diet, $20 \%$ sucrose, $10 \%$ egg yolk powder and $10 \%$ lard for 6 weeks, and were then intraperitoneally injected with $35 \mathrm{mg} / \mathrm{kg}$ streptozotocin (STZ; Shanghai Yuanye Biotechnology Co., Ltd.), which was dissolved in a $0.1 \mathrm{M}$ citric acid-sodium citrate buffer ( $\mathrm{pH} 4.4)$. Rats in the normal control group were maintained on the standard diet for 6 weeks and were then given the same amount of vehicle $(0.1 \mathrm{M}$ citric acid-sodium citrate buffer, $\mathrm{pH}$ 4.4) via an intraperitoneal injection. After $72 \mathrm{~h}$ and 7 days, the levels of fasting blood glucose (FBG) in the tail vein were measured with a portable glucometer (GA-3 Sannuoyi accurate blood glucometer; Changsha Sannuo Biosensing Co., Ltd.). The success of the diabetic rats was based on FBG concentration $\geq 16.7 \mathrm{mmol} / \mathrm{l}(18,19)$.

Then, 1 week after STZ injection, diabetic rats were randomly divided into three subgroups according to different treatment methods ( $\mathrm{n}=6 / \mathrm{each}$ subgroup): Diabetic model group, AS-IV treatment group and Met treatment group, and were still fed a HFD. Rats in the AS-IV and Met treatment groups were treated with an intragastric administration of AS-IV $(80 \mathrm{mg} / \mathrm{kg})$ and Met $(200 \mathrm{mg} / \mathrm{kg})$ daily for 8 weeks, respectively. The normal control group and model group rats were treated with an equal volume of vehicle $\left(0.5 \% \mathrm{CMC}-\mathrm{Na}^{+}\right.$ aqueous solution) daily for 8 weeks. After 8 weeks of intervention, rats were fasted overnight for $12 \mathrm{~h}$ and anaesthetised with pentobarbital sodium $(30 \mathrm{mg} / \mathrm{kg})$. Blood samples $(8-10 \mathrm{ml} / \mathrm{each}$ rat) were collected via the abdominal aorta for further analysis when the rats lost consciousness, and then the rats were euthanized by exsanguination after anaesthesia. Liver tissues were removed and weighed immediately for further analysis.

Measurement of food intake, water intake, urine volume, body weight and FBG. During the experiment, the general conditions of the rats were observed, such as food intake, water intake, urine volume and body weight. The food intake, water intake and urine volume of rats within $24 \mathrm{~h}$ were measured every 4 weeks. Additionally, at a fixed time (every Saturday at $8 \mathrm{am}$ ), the body weight of the rats was measured 1 a week, and the levels of FBG from the tail vein after fasting for $6 \mathrm{~h}$ in each group were measured every 2 weeks from 0 to 8 weeks of administration using a portable glucometer.

Oral glucose tolerance test (OGTT). The OGTT was performed on rats that were fasted overnight for $12 \mathrm{~h}$ after the last administration (the normal control group, diabetic model group, AS-IV treatment group and Met treatment group were treated with $\mathrm{CMC}-\mathrm{Na}^{+}$aqueous solution, $\mathrm{CMC}-\mathrm{Na}^{+}$aqueous solution, AS-IV and Met, respectively). Rats in each group were given glucose at a dose of $2 \mathrm{~g} / \mathrm{kg}$ by gavage. Blood glucose levels were measured using the portable glucometer via the detection of blood samples collected from the tail vein at 0 (before gavage), 30,60 and $120 \mathrm{~min}$ after gavage. The data were plotted curves of the blood glucose concentrations over time, and the area under the curve (AUC) was calculated.

Measurement of biochemical analyses. Blood samples were centrifuged at $2,000 \mathrm{x}$ g for $10 \mathrm{~min}$ at $4^{\circ} \mathrm{C}$ for analysis. Serum levels of alanine aminotransferase (ALT; cat. no. C009-2-1), aspartate aminotransferase (AST; cat. no. C010-2-1), total cholesterol (TC; cat. no. A111-1), triglyceride (TG; cat. no. A110-1-1), high-density lipoprotein cholesterol (HDL-C; cat. no. A112-1-1), low-density lipoprotein cholesterol (LDL-C; cat. no. A113-1-1) and glycosylated serum protein (GSP; cat. no. A037-2) were determined using the corresponding kits (Nanjing Jiancheng Bioengineering Institute) according to the manufacturer's instructions. The levels of TNF- $\alpha$ (cat. no. MM-0180R1), IL-6 (cat. no. MM-0190R2) and fasting insulin (FINS; cat. no. MM-0587R1) in serum 
were measured with their commercial ELISA kits (Jiangsu Enzyme Free Experimental Co., Ltd.) according to the manufacturer's instructions. The homeostasis model assessment of insulin resistance (HOMA-IR), which indicates the degree of IR, was calculated according to the following formula: HOMA-IR=(FBGxFINS)/22.5 (20).

Detection of liver histology. Liver specimens were fixed in $4 \%$ paraformaldehyde at room temperature for 24-48 h, dehydrated, embedded in paraffin blocks and cut into 5- $\mu \mathrm{m}$ sections. Liver sections were dewaxed in xylene, rehydrated in graded alcohol series and rinsed under running water. Subsequently, sections were stained with H\&E (haematoxylin 3-5 min and eosin $30 \mathrm{sec}$, both at room temperature) or Masson's trichrome staining (cat. no. G1340; Beijing Solarbio Science \& Technology Co., Ltd.; haematoxylin $3 \mathrm{~min}$, ponceau acid fuchsin 5-10 min, phosphomolybdic acid 1-3 min and aniline blue 3-6 min, both at room temperature), and histopathological changes were examined under a light microscope (Olympus IX71; Olympus Corporation; magnification, $\mathrm{x} 400$ ). The intensity of positive areas (Masson's staining) was analysed using Image-Pro Plus 6.0 analysis software (Media Cybernetics, Inc.) to assess liver fibrosis.

Lipid deposition was assessed via Oil red $\mathrm{O}$ staining in the liver. Briefly, liver specimens were embedded in optimal cutting temperature compound and sliced with a frozen slicer (Leica CM3050; Leica Microsystems GmbH) at a thickness of $10-\mu \mathrm{m}$. Liver slices were fixed with $10 \%$ formalin at room temperature for $15 \mathrm{~min}$, stained with Oil red $\mathrm{O}$ solution at room temperature for 8-10 min and observed under a light microscope (Olympus IX71; Olympus Corporation). The intensity of positive areas from $\geq 5$ random fields (magnification, $x 400$ ) in each section was quantified using Image-Pro Plus 6.0 analysis software to assess lipid deposition.

Immunohistochemistry. Liver tissues were fixed in $4 \%$ paraformaldehyde for $24 \mathrm{~h}$ at room temperature and embedded in liquid paraffin at room temperature, and after waiting to solidify, these were cut into 5- $\mu \mathrm{m}$ sections. After dewaxing and rehydration, the liver sections were placed in sodium citrate buffer (pH 6.0) in a microwave oven for antigen retrieval (boiling for 5-10 min, fire ceased for $3 \mathrm{~min}$, boiling for 5-10 min). After natural cooling, the sections were incubated in $3 \% \mathrm{H}_{2} \mathrm{O}_{2}$ for $25 \mathrm{~min}$ to inhibit endogenous peroxidase activity and were then blocked with 3\% BSA (cat. no. 4240GR100; BioFroxx GmbH) at $37^{\circ} \mathrm{C}$ for $30 \mathrm{~min}$. Immunohistochemistry was performed using Beclin1 (cat. no. AF5128; 1:100; Affinity Biosciences), P62 (cat. no. AF5384; 1:100; Affinity Biosciences) and LC3 (cat. no. AF5402; 1:100; Affinity Biosciences) primary antibodies at $4{ }^{\circ} \mathrm{C}$ overnight. The sections were then treated with HRP-labelled goat anti-rabbit secondary antibodies (cat. no. S0001; 1:500; Affinity Biosciences) at $37^{\circ} \mathrm{C}$ for $50 \mathrm{~min}$. Finally, DAB chromogen (cat. no. G1211; Wuhan Servicebio Technology Co., Ltd.) was added before counterstaining with haematoxylin at room temperature for $3 \mathrm{~min}$. The intensity of positive areas from $\geq 5$ random fields (magnification, $x 400$ ) in each section was quantified using Image-Pro Plus 6.0 analysis software.

Western blotting. Liver tissues were collected, homogenised and then lysed in ice-cold RIPA lysis buffer (cat. no. P0013B;
Beyotime Institute of Biotechnology) containing protease inhibitors and phosphatase inhibitors to extract the total proteins. The concentration of total proteins was determined using the enhanced BCA protein assay kit (cat. no. P0010S; Shanghai Beyotime Biotechnology Co., Ltd.) according to the manufacturer's instructions. The same amount of protein (30 $\mu \mathrm{g}$ ) was separated by $8-15 \%$ SDS-PAGE and transferred to PVDF membranes (EMD Millipore). Then, the membranes were blocked with $5 \%$ fat-free milk in TBS- $0.05 \%$ Tween-20 (TBST) buffer at room temperature for $2 \mathrm{~h}$ and incubated overnight at $4{ }^{\circ} \mathrm{C}$ with primary antibodies against AMPK (cat. no. AF6423; 1:1,000; Affinity Biosciences), phosphorylated (p)-AMPK (cat. no. BS4010; 1:1,000; Bioworld Technology, Inc.), mTOR (cat. no. 66888-1-1g; 1:5,000; ProteinTech Group, Inc.), p-mTOR (cat. no. BS4706; 1:1,000; Bioworld Technology, Inc.), Beclin1 (cat. no. WL02508; 1:1,000; Wanleibio Co., Ltd.), P62 (cat. no. WL02385; 1:500; Wanleibio Co., Ltd.), LC3 (cat. no. 14600-1-AP; 1:1,000; ProteinTech Group, Inc.), TNF- $\alpha$ (cat. no. AF7014; 1:500; Affinity Biosciences), IL-6 (cat. no. BS6419; 1:1,000; Bioworld Technology, Inc.), heme oxygenase (HO)-1 (cat. no. ab13243; $1: 2,000 ;$ Abcam) and $\beta$-actin (cat. no. AF7019; 1:5,000; Affinity Biosciences). Subsequently, the membranes were washed with TBST buffer three times for $10 \mathrm{~min}$ and then incubated for $1 \mathrm{~h}$ at room temperature with the corresponding HRP-conjugated secondary antibody (cat. no. S0001; 1:10,000; Affinity Biosciences). An ECL reagent (Bridgen Co., Ltd.: http://www.bridgen.cn) was used to visualise the blotted proteins using a Chemi Doc TMMP Imaging system (Bio-Rad Laboratories, Inc.). The density of the target bands was analysed semi-quantitatively using Image J 1.45 s software (National Institutes of Health) and normalised with $\beta$-actin as the internal control.

Transmission electron microscopy detection. Liver specimens were cut into small pieces $\left(1 \mathrm{~mm}^{3}\right)$, and immediately fixed with $2.5 \%$ glutaraldehyde for $2 \mathrm{~h}$ at $4^{\circ} \mathrm{C}$. After washing with PBS, the tissues were post-fixed in $1 \%$ osmium tetroxide for $2 \mathrm{~h}$ at room temperature, dehydrated and then embedded in Epon 812. Ultrathin sections (thickness, $70 \mathrm{~nm}$ ) were cut with an ultramicrotome (Leica UC7; Leica Microsystems GmbH) and double-stained with uranyl acetate and lead citrate at room temperature for $15 \mathrm{~min}$. Ultrastructural images were viewed and imaged using a transmission electron microscope (HT 7700; Hitachi, Ltd.; magnification, x5,000).

Statistical analysis. Data are presented as the mean \pm SD of $\geq 3$ independent experiments. SPSS 16.0 statistical software (SPSS, Inc.) was used to compare differences among groups via a one-way ANOVA followed by Tukey's test. Mixed two-way ANOVA and Bonferroni tests were used to measure differences over time and between-subject comparisons of treatment groups. $\mathrm{P}<0.05$ was considered to indicate a statistically significant difference.

\section{Results}

Effects of AS-IV on food intake, water intake, urine volume and body weight in T2DM rats. As shown in Fig. 1A-C, at the end of the 4 and 8 th weeks of the experiment, the food intake, 

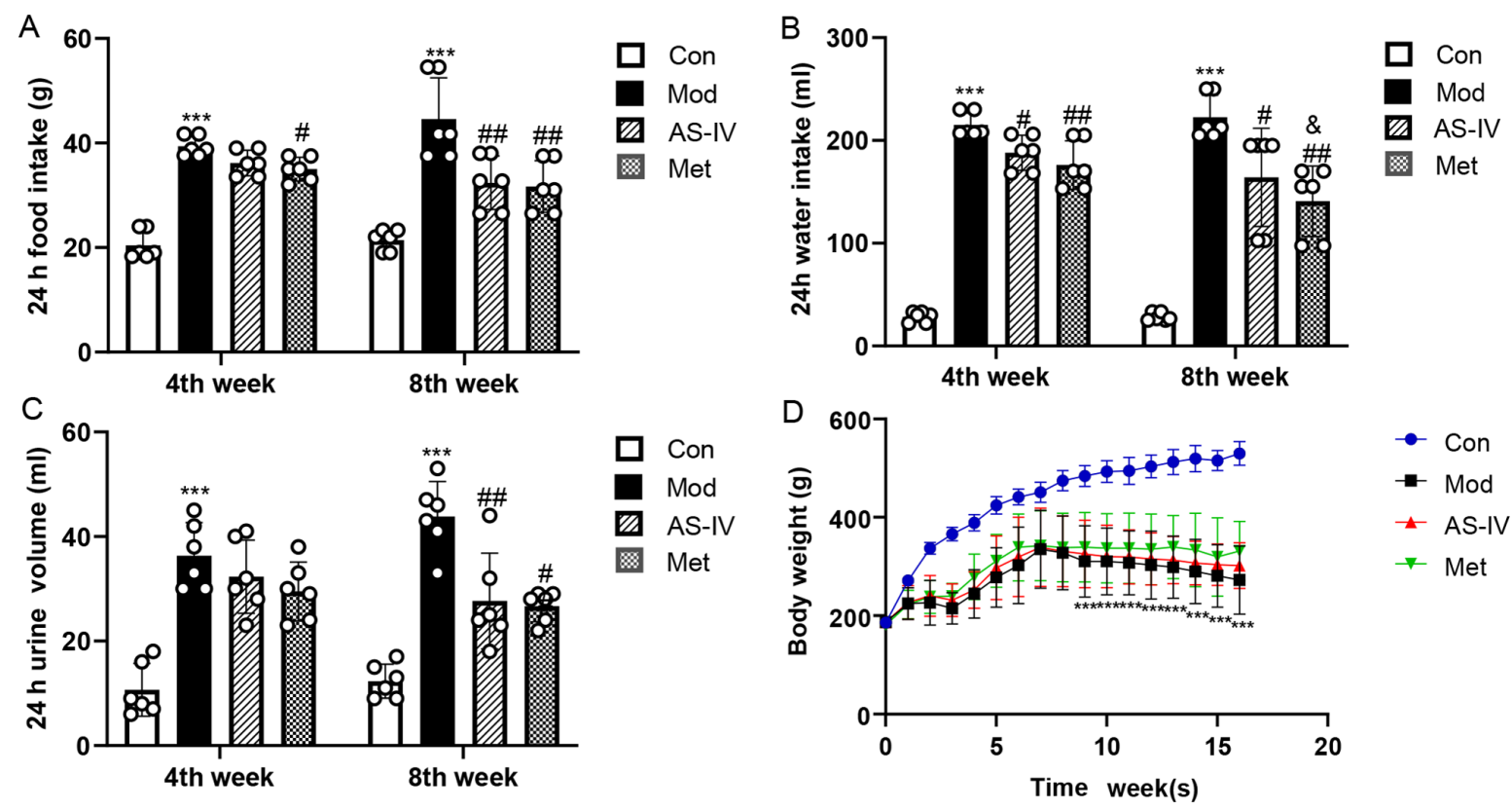

Figure 1. Effects of AS-IV on food intake, water intake, urine volume and body weight in T2DM rats. The (A) $24 \mathrm{~h}$ food intake, (B) $24 \mathrm{~h}$ water intake and (C) $24 \mathrm{~h}$ urine volume at the end of the 4 and 8th weeks after administration. (D) Body weight from 0-16 weeks (the administration was started on the 9th week). Data are presented as the mean $\pm \mathrm{SD}, \mathrm{n}=6$. ${ }^{* * *} \mathrm{P}<0.001$ vs. control group; ${ }^{\sharp} \mathrm{P}<0.05,{ }^{\# \#} \mathrm{P}<0.01$ vs. model group; ${ }^{\circledR} \mathrm{P}<0.05$ vs. 4th week. Con, Control; Mod, Model; AS-IV, Astragaloside IV (80 mg/kg); Met, Metformin (200 mg/kg).

water intake and urine volume were significantly increased in the model group compared with the control group $(\mathrm{P}<0.001)$, while AS-IV and Met treatment significantly decreased these indicators compared with the model group $(\mathrm{P}<0.05$ or $\mathrm{P}<0.01)$, especially at the end of the 8th week. Furthermore, during the experiment, the body weight of the model group was significantly lower compared that of the control group $(\mathrm{P}<0.001$; Fig. 1D), while AS-IV and Met treatment notably inhibited the weight loss of the T2DM rats, but the difference was not statistically significant ( $P>0.05$; Fig. 1D). These results support a feasible role for AS-IV to improve food intake, water intake, urine volume and body weight in T2DM rats.

Effects of AS-IV on liver function in T2DM rats. ALT, AST and the liver index of liver weight to body weight were detected to investigate the effect of AS-IV on liver function in T2DM rats. ALT and AST, which are sensitive markers of liver function, are released into the blood due to the increased permeability of the cell membrane when liver cells are damaged, resulting in increased levels of ALT and AST in serum (21). The serum levels of ALT and AST in the model group were significantly higher compared with those in the control group, but were reversed by treatment with AS-IV and Met $(\mathrm{P}<0.01$ or $\mathrm{P}<0.001$; Fig. 2D and E). It has been reported that the liver index, another indicator to evaluate liver function, is abnormally increased in diabetes (22). It was found that the liver index in the model group was significantly increased compared with that of the control group, but was significantly reduced by treatment with AS-IV and Met $(\mathrm{P}<0.01$ or $\mathrm{P}<0.001$; Fig. $2 \mathrm{~F})$.

To further observe liver histopathology, H\&E staining was performed. As presented in Fig. 2A, neatly arranged hepatocytes without steatosis were observed in the control group. In the model group, disorganised hepatocytes and lipid droplets were notably increased. However, AS-IV and Met treatment showed marked improvements, such as reduced liver lipid droplets and hepatic steatosis. Additionally, hepatic fibrosis was detected using Masson staining. Compared with the control group, the model group showed significant hepatic fibrosis with enlarged blue areas ( $\mathrm{P}<0.001$; Fig. $2 \mathrm{~B}$ and $\mathrm{C})$, suggesting collagen deposition in the liver of T2DM rats. By contrast, compared with the model group, the collagen deposition was significantly improved by the administration of AS-IV and Met ( $\mathrm{P}<0.01$; Fig. 2B and $\mathrm{C})$. These data suggest that AS-IV treatment improves liver function in T2DM rats.

Effects of AS-IV on glucose homeostasis in T2DM rats. To determine whether AS-IV improves glucose homeostasis in T2DM rats, GSP, FBG, FINS and HOMA-IR in serum were examined. In the model group, the levels of FBG, FINS, HOMA-IR and GSP were significantly increased, but AS-IV and Met treatment reversed the elevated levels of these indexes $(\mathrm{P}<0.05, \mathrm{P}<0.01$ or $\mathrm{P}<0.001$; Fig. 3A-D). Moreover, FBG and OGTT levels were examined by taking blood from the tail vein. As presented in Fig. 3E, a significant increase in FBG level was observed in the model group, especially at 6-8 weeks $(\mathrm{P}<0.01$ or $\mathrm{P}<0.001)$. It was found that AS-IV treatment resulted in no hypoglycaemic activity in the first 2 weeks, whereas long-term treatment with AS-IV (4-8 weeks), especially at 6-8 weeks, had a hypoglycaemic activity compared with the model group $(\mathrm{P}<0.001)$. In addition, the AUC of the OGTT in the model group was higher compared with that in the control group, but the elevations were significantly reversed by AS-IV and Met treatment $(\mathrm{P}<0.001$; Fig. 3F). Collectively, these results indicated that AS-IV could improve glucose homeostasis in T2DM rats.

Effects of AS-IV on dyslipidaemia and liver lipid deposition in T2DM rats. To determine whether AS-IV ameliorates 

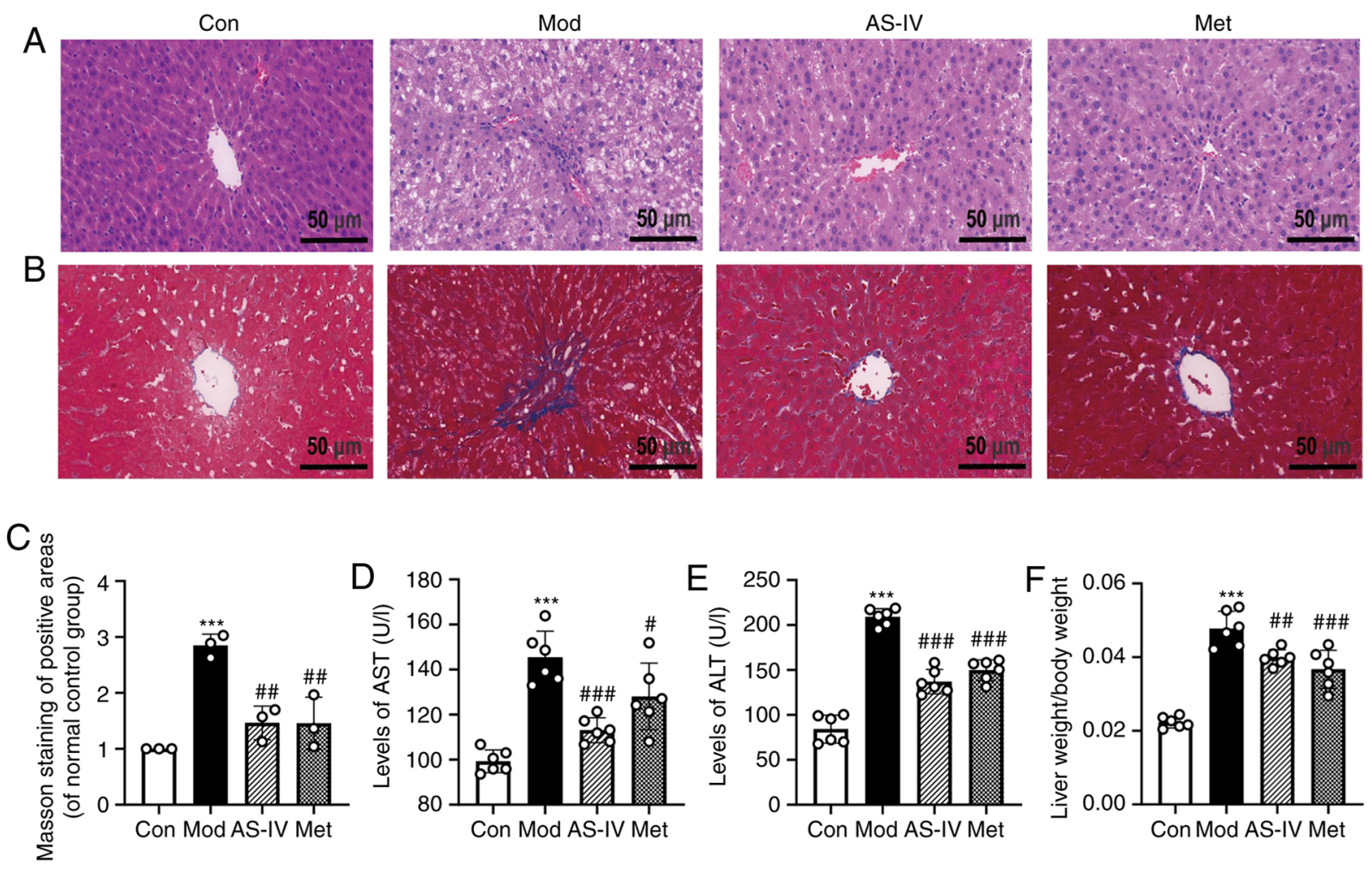

Figure 2. AS-IV attenuates liver function in T2DM rats. (A) H\&E staining of liver tissues (magnification, x400). (B) Masson staining of liver tissues (magnification, x400). (C) Quantitative analysis of positive areas of Masson staining was normalised to the normal control group. (D) AST and (E) ALT levels in serum. (F) Ratio of liver weight to body weight. Data are presented as the mean $\pm \mathrm{SD}, \mathrm{n}=6$. ${ }^{* * *} \mathrm{P}<0.001$ vs. control group; ${ }^{\#} \mathrm{P}<0.05$, ${ }^{\# \#} \mathrm{P}<0.01$, ${ }^{\# \#} \mathrm{P}<0.001$ vs. model group. Con, Control; Mod, Model; AS-IV, Astragaloside IV (80 mg/kg); Met, Metformin (200 mg/kg); AST, aspartate aminotransferase; ALT, alanine aminotransferase.

dyslipidaemia in T2DM rats, the levels of TG, TC, LDL-C and HDL-C were detected in serum. Compared with the control group, the TG and TC levels were significantly increased in the model group, but the HDL-C level was markedly decreased $(\mathrm{P}<0.001$; Fig. 4C, D and F), indicating that T2DM may cause dyslipidaemia. By contrast, AS-IV and Met treatment significantly lowered TG and TC levels, and increased HDL-C level $(\mathrm{P}<0.05$ or $\mathrm{P}<0.001$; Fig. 4C, D and F). However, there was no significant change in LDL-C levels (Fig. 4E).

$H \& E$ staining revealed a large number of vacuoles in the liver of the model group, while AS-IV and Met treatment induced significant improvements with reduced vacuoles (Fig. 2A). To further confirm these findings, Oil red $\mathrm{O}$ staining was performed in the liver to assess lipid deposition. As illustrated in Fig. 4A and B, the lipid deposition in the model group was significantly increased compared with the control group $(\mathrm{P}<0.001)$, while the degree of lipid deposition was significantly improved after AS-IV and Met administration $(\mathrm{P}<0.001)$. These results demonstrate that AS-IV treatment improves dyslipidaemia and liver lipid deposition in T2DM rats.

Effects of AS-IV on inflammation and oxidative stress in the liver of T2DM rats. To examine the level of inflammation in the liver, the levels of pro-inflammatory cytokines TNF- $\alpha$ and IL- 6 were measured. The serum levels of TNF- $\alpha$ and IL-6 in the model group were significantly higher compared with those in the control group $(\mathrm{P}<0.001$; Fig. 5A and $\mathrm{B})$. After the administration of AS-IV and Met, the serum levels of inflammatory cytokines were significantly reduced $(\mathrm{P}<0.001$; Fig. 5A and B). Similarly, the western blotting results demonstrated that the expression levels of TNF- $\alpha$ and IL- 6 in the liver of the model group were increased significantly compared with the control group $(\mathrm{P}<0.001$; Fig. 5C-E). Moreover, AS-IV and Met treatment significantly decreased these indexes compared with the model group $(\mathrm{P}<0.05$ or $\mathrm{P}<0.01$; Fig. $5 \mathrm{C}-\mathrm{E})$.

$\mathrm{HO}-1$ is an important antioxidant enzyme involved in numerous biological processes. After oxidative stress and cell damage, HO-1 expression can be upregulated $(23,24)$. It has been reported that the protein expression level of HO-1 was increased under the conditions of diabetes and other diseases, and its expression was further enhanced when the administration of chlorogenic acid and biochanin A treatment improved oxidative stress $(23,24)$. The current study further examined the expression level of the oxidative stress protein $\mathrm{HO}-1$ in the liver via western blotting. As shown in Fig. 5C and F, the expression level of HO-1 was increased in the model group compared with the control group $(\mathrm{P}<0.05)$. However, compared with the model group, AS-IV and Met treatment significantly upregulated the expression level of HO-1 $(\mathrm{P}<0.05)$. The results suggest that AS-IV suppresses the inflammation and oxidative stress in the liver of T2DM rats.

AS-IV activates autophagy in the liver of T2DM rats. Previous studies have revealed that autophagy can ameliorate IR, dyslipidaemia, oxidative stress and inflammation (8-11). Based on previous research, the current study focused on autophagy. 
A

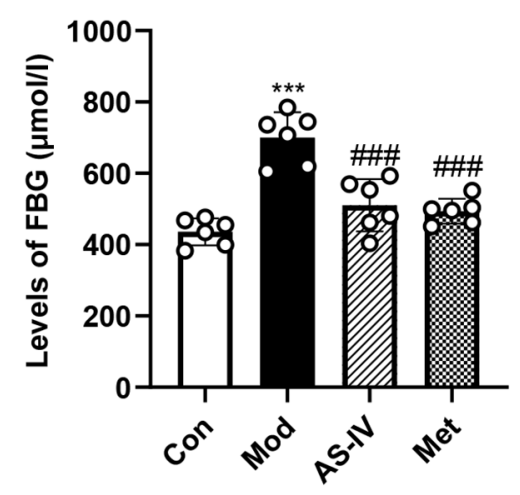

D

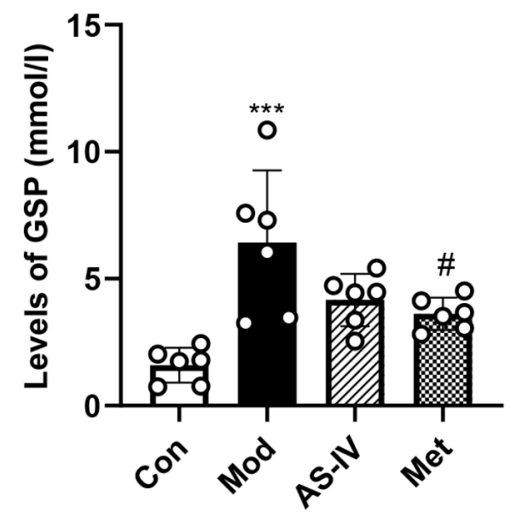

$\mathrm{F}$

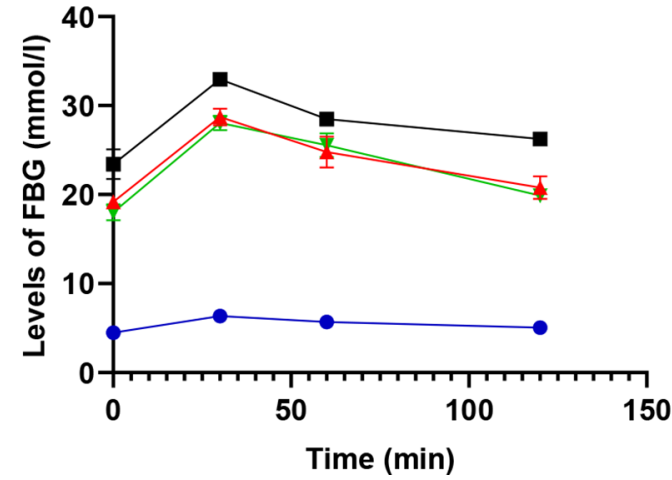

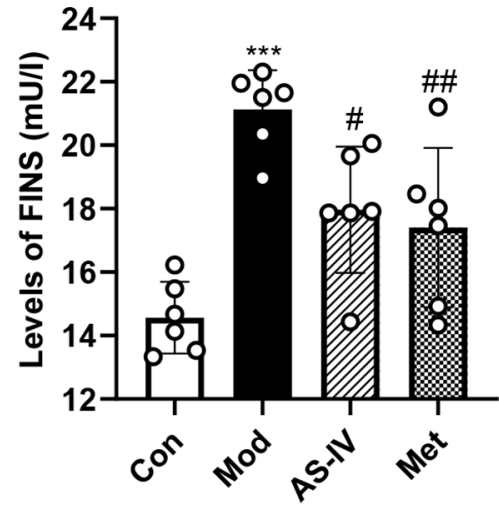
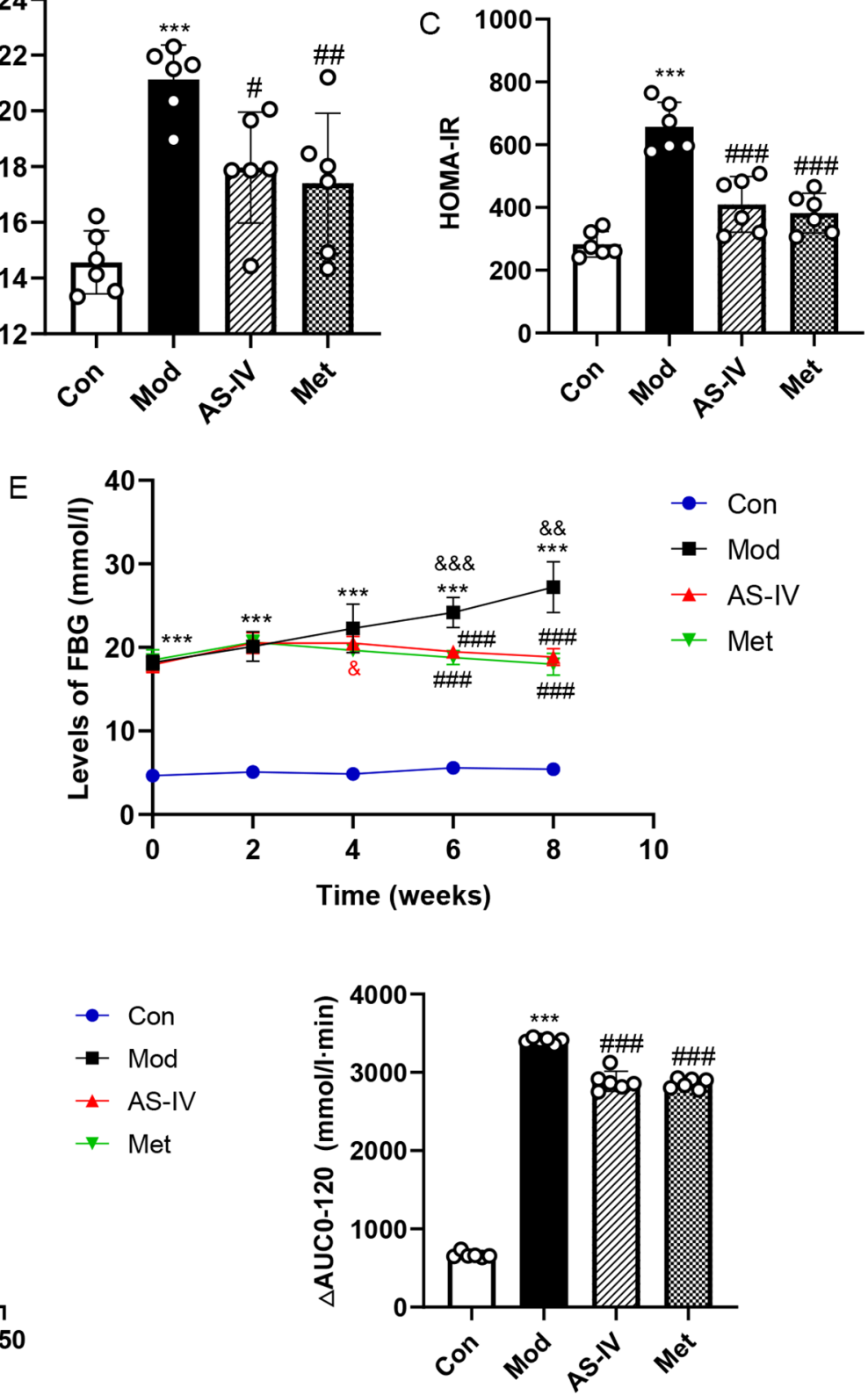

Figure 3. AS-IV maintains glucose homeostasis in T2DM rats. (A) FBG and (B) FINS levels in serum. (C) HOMA-IR. (D) GSP levels in serum. (E) FBG levels from $0-8$ weeks after intervention. $(\mathrm{F})$ Oral glucose tolerance test results and AUC. Data are presented as the mean $\pm \mathrm{SD}, \mathrm{n}=6$. ${ }^{* * *} \mathrm{P}<0.001 \mathrm{vs}$. control group; ${ }^{\#} \mathrm{P}<0.05,{ }^{\# \#} \mathrm{P}<0.01,{ }^{\# \#} \mathrm{P}<0.001$ vs. model group; ${ }^{\&} \mathrm{P}<0.05$, ${ }^{\&} \mathrm{P}<0.01$, \&\&\& $\mathrm{P}<0.001$ vs. week 0. Con, Control; Mod, Model; AS-IV, Astragaloside IV (80 mg/kg); Met, Metformin (200 mg/kg); FBG, fasting blood glucose; GSP, glycosylated serum protein; FINS, fasting insulin; HOMA-IR, homeostasis model assessment of insulin resistance; AUC, area under the curve.

To analyse the effect of AS-IV on autophagy under diabetic liver injury conditions, liver autophagy was examined using western blotting and immunohistochemistry to detect the protein levels of Beclin1, LC3 and P62 in the liver. As presented in Fig. 6A, B, E and F, the accumulation of Beclin1, an essential autophagic protein in the initial stages of autophagy (25), was decreased in the model group $(\mathrm{P}<0.001)$, but it was significantly increased in the AS-IV and Met treatment groups $(\mathrm{P}<0.01$ or $\mathrm{P}<0.001)$. Moreover, the transformation from LC3I to LC3II, a marker of autophagy (26), was decreased in the model group, but was significantly increased by AS-IV and Met treatment $(\mathrm{P}<0.05$ or $\mathrm{P}<0.001$; Fig. $6 \mathrm{~A}, \mathrm{C}, \mathrm{E}$ and $\mathrm{G})$. $\mathrm{P} 62$ is involved in the degradation of the proteasome and can reflect autophagy activity (27). It was found that P62 expression was elevated in the model group, but it was significantly decreased after treatment with AS-IV and Met $(\mathrm{P}<0.05$ or $\mathrm{P}<0.001$; Fig. 6A, D, E and H).

The autophagosomes in the liver were examined using a transmission electron microscope to determine the occurrence of autophagy. Compared with the control group, the autophagosomes in the model group were inhibited, while AS-IV and Met treatment increased autophagosome formation (Fig. 7). These results indicate that AS-IV enhances liver autophagy in T2DM rats.

AS-IV activates the AMPK/mTOR pathway in the liver of T2DM rats. To evaluate the potential mechanism of AS-IV activation of autophagy, the AMPK/mTOR pathway, a major 
A

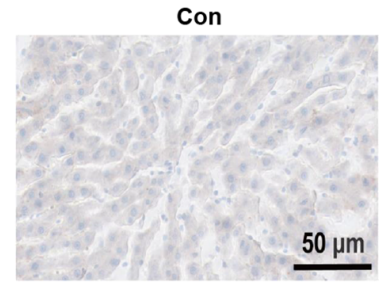

AS-IV

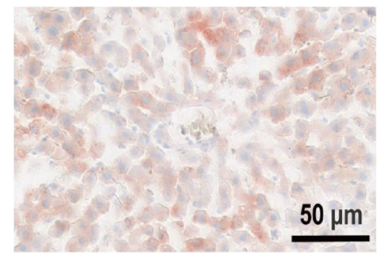

C

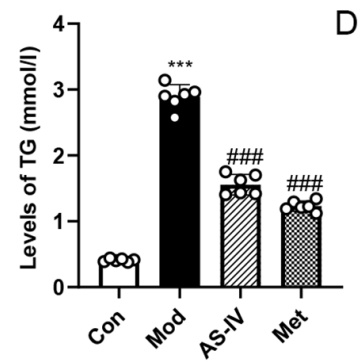

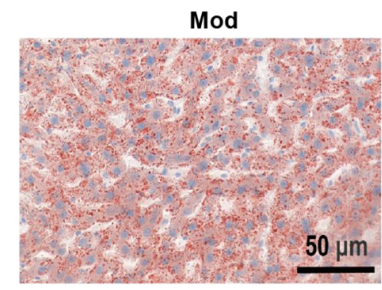

Met

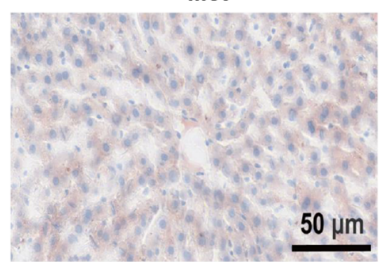

B

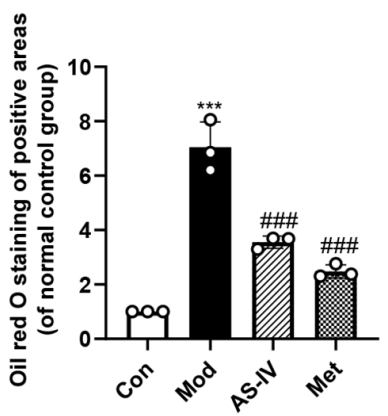

Figure 4. AS-IV improves dyslipidaemia and liver lipid deposition in T2DM rats. (A) Oil red O staining of liver tissues (magnification, $\mathrm{x} 400$ ). (B) Quantitative analysis of positive areas of Oil red O staining was normalised to the normal control group. (C) TG, (D) TC, (E) HDL-C and (F) LDL-C levels in serum. Data are presented as the mean $\pm \mathrm{SD}, \mathrm{n}=3-6 .{ }^{* * *} \mathrm{P}<0.001$ vs. control group; ${ }^{*} \mathrm{P}<0.05,{ }^{\# \# \#} \mathrm{P}<0.001$ vs. model group. Con, Control; Mod, Model; AS-IV, Astragaloside IV (80 mg/kg); Met, Metformin (200 mg/kg); TC, total cholesterol; TG, triglyceride; HDL-C, high-density lipoprotein cholesterol; LDL-C, low-density lipoprotein cholesterol.

A

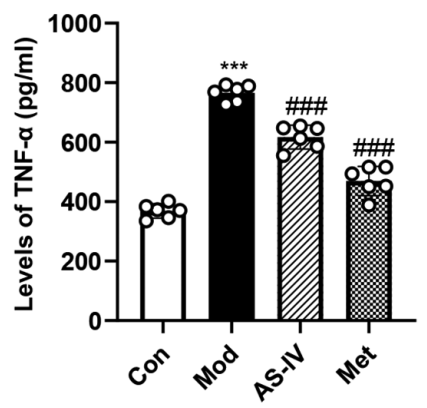

D

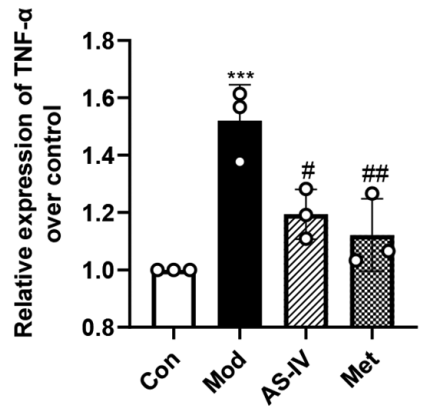

$\mathrm{B}$

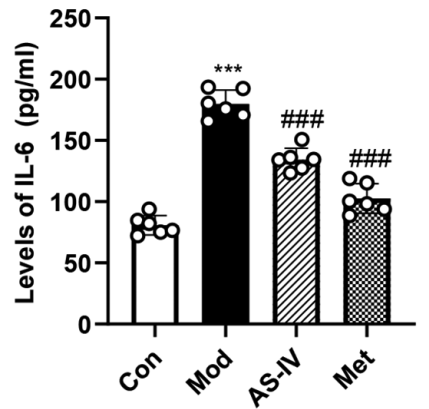

E

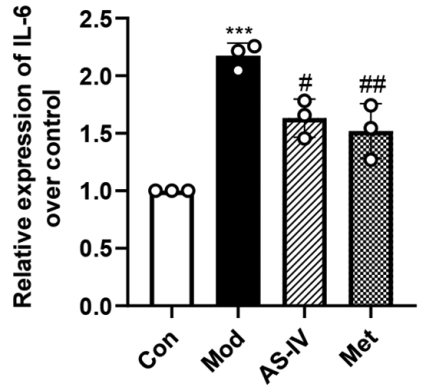

C
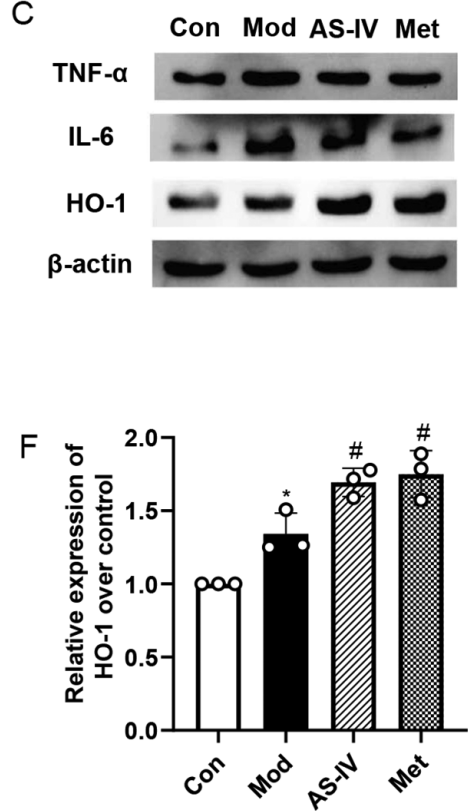

Figure 5. AS-IV ameliorates inflammation and oxidative stress in the liver of T2DM rats. (A) TNF- $\alpha$ and (B) IL-6 levels in serum. (C) Protein expression levels of TNF- $\alpha$, IL-6 and HO1 in the liver tissues were detected via western blotting. Semi-quantitative analysis of (D) TNF- $\alpha$, (E) IL-6 and (F) HO-1 was normalised to $\beta$-actin. Data are presented as the mean $\pm \mathrm{SD}, \mathrm{n}=3$. ${ }^{* * * *} \mathrm{P}<0.001$ vs. control group; ${ }^{\#} \mathrm{P}<0.05,{ }^{\# \#} \mathrm{P}<0.01$, ${ }^{\# \# \#} \mathrm{P}<0.001$ vs. model group. Con, Control; Mod, Model; AS-IV, Astragaloside IV (80 mg/kg); Met, Metformin (200 mg/kg); HO-1, heme oxygenase-1.

pathway that regulates liver autophagy (28), was examined in the liver. As presented in Fig. 8A-C, the expression level of p-AMPK was significantly downregulated and the expression level of p-mTOR was significantly upregulated in the model group compared with the control group $(\mathrm{P}<0.01$ or $\mathrm{P}<0.001)$; there was no significant change in AMPK and mTOR 
A
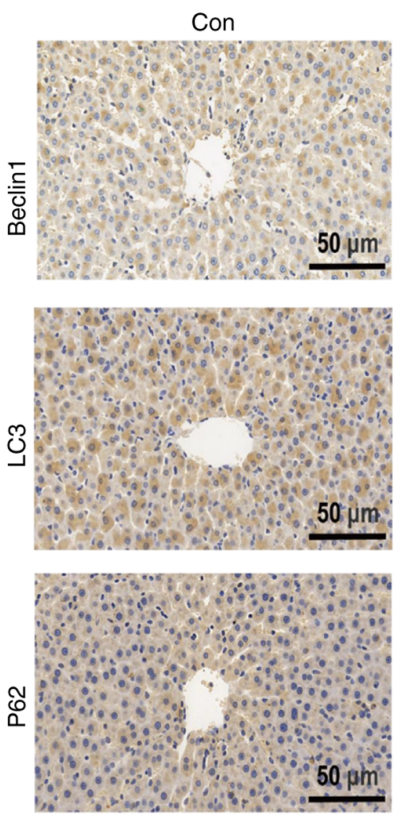
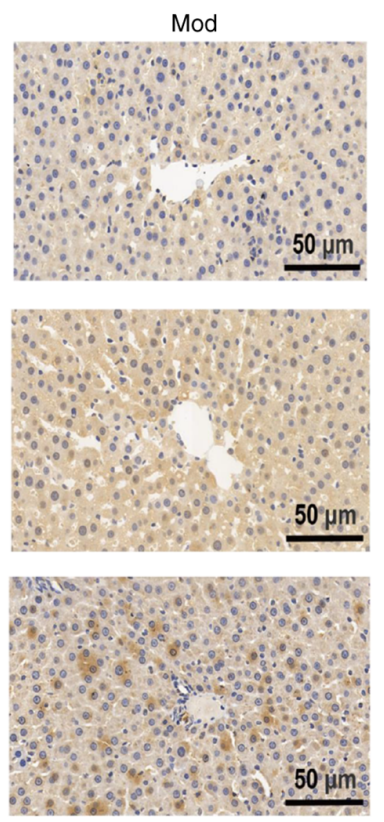
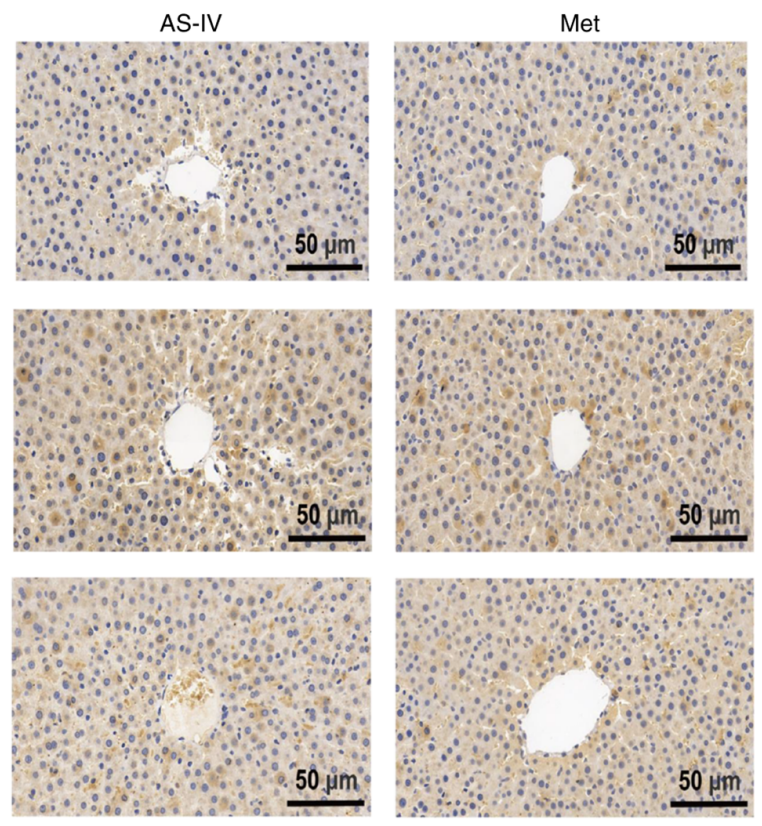

E
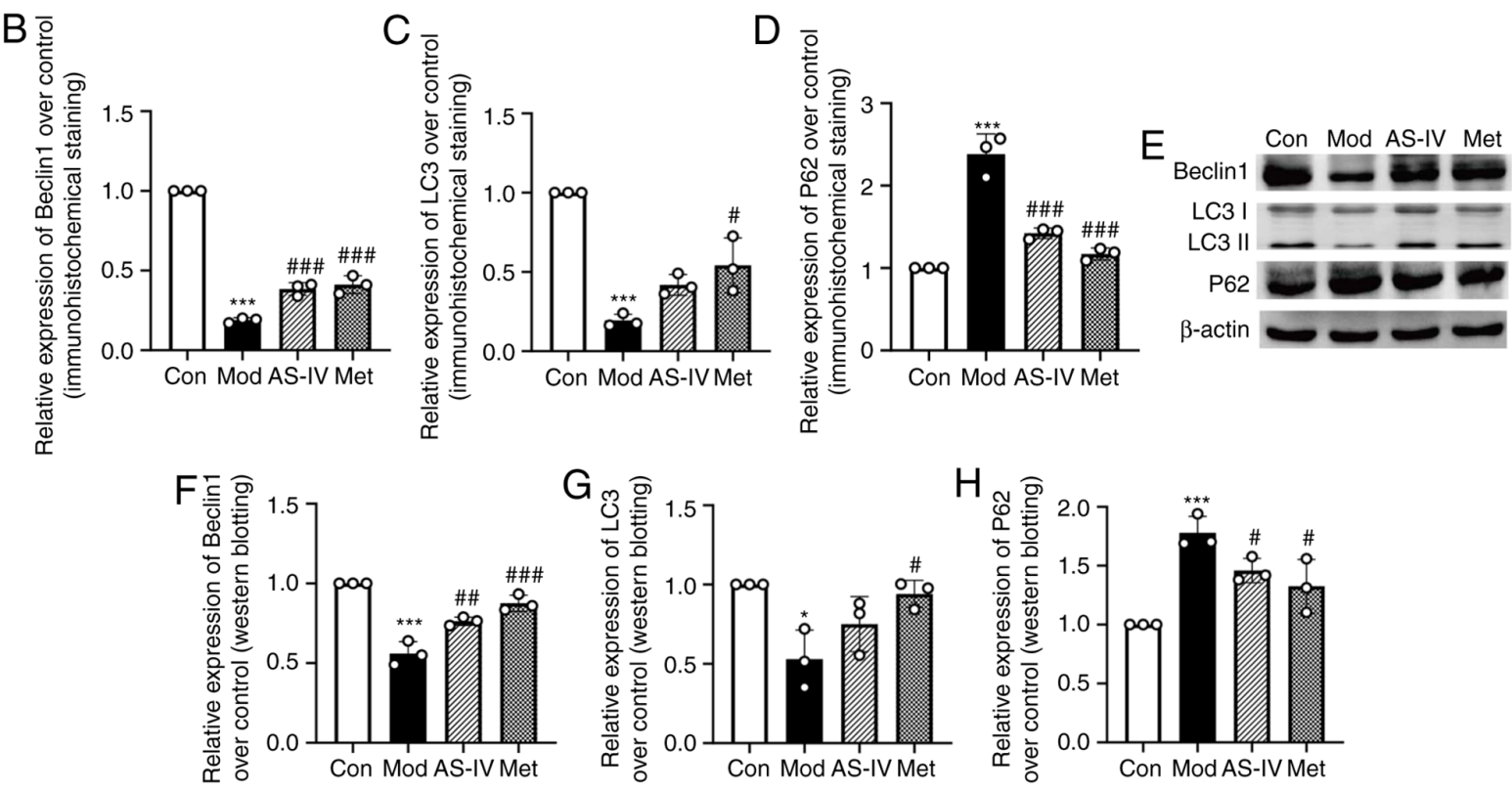

Figure 6. AS-IV activates the suppressed autophagy in the liver of T2DM rats. (A) Representative images of Beclin1, LC3 and P62 (immunohistochemical staining; magnification, x400). Quantitative analysis of (B) Beclin1, (C) LC3 and (D) P62 were normalized to the normal control group. (E) Protein expression levels of Beclin1, LC3 and P62 in the liver tissues were detected via western blotting. Semi-quantitative analysis of (F) Beclin1, (G) LC3 and (H) P62 was normalised to $\beta$-actin. Data are presented as the mean $\pm \mathrm{SD}, \mathrm{n}=3 .{ }^{*} \mathrm{P}<0.05,{ }^{* * *} \mathrm{P}<0.001$ vs. control group; ${ }^{\#} \mathrm{P}<0.05,{ }^{\# \#} \mathrm{P}<0.01,{ }^{\# \# \#} \mathrm{P}<0.001$ vs. model group. Con, Control; Mod, Model; AS-IV, Astragaloside IV ( $80 \mathrm{mg} / \mathrm{kg})$; Met, Metformin $(200 \mathrm{mg} / \mathrm{kg})$.

expression. However, the suppressed AMPK/mTOR pathway in the model group was significantly reversed by AS-IV and Met treatment, as evidenced by the significantly elevated p-AMPK/AMPK ratio $(\mathrm{P}<0.01$ or $\mathrm{P}<0.001)$ and decreased $\mathrm{p}$-mTOR/mTOR $(\mathrm{P}<0.05$ or $\mathrm{P}<0.01)$ ratio in the AS-IV and Met treatment groups. These data suggest that AS-IV may enhance autophagy via the activation of the AMPK/mTOR pathway in the liver of T2DM rats.

\section{Discussion}

The present study provided evidence that AS-IV treatment ameliorated diabetic liver injury in T2DM rats. Furthermore, it was confirmed that autophagy served a crucial role in diabetic liver injury in T2DM rats, which is also closely associated with the AMPK/mTOR signalling pathway. Based on the protective effect of AS-IV on diabetic liver injury by promoting autophagy, the current research indicated that AS-IV administration may provide a potentially effective treatment strategy for diabetic liver injury in T2DM.

Diabetic liver injury is one of the complications in patients with T2DM (5). At present, there is no effective treatment method for diabetic liver injury, and so the effective functional ingredients from plants have become a new treatment method (29-31). AS-IV is the main active ingredient of medicinal plant Astragalus membranaceus, which has been reported 

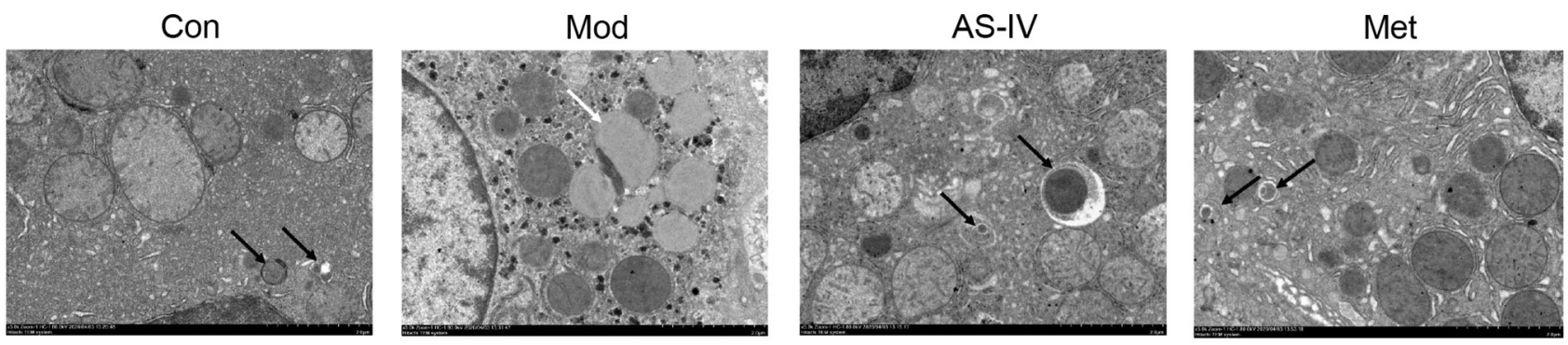

Figure 7. Transmission electron microscopy images showing the ultrastructure of lipids (white arrows) and autophagosomes (black arrows). $\mathrm{n}=3$ for each group. Con, Control; Mod, Model; AS-IV, Astragaloside IV (80 mg/kg); Met, Metformin (200 mg/kg).

A

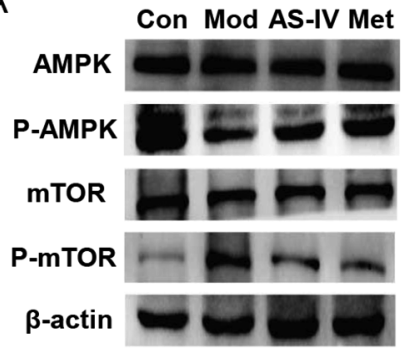

B

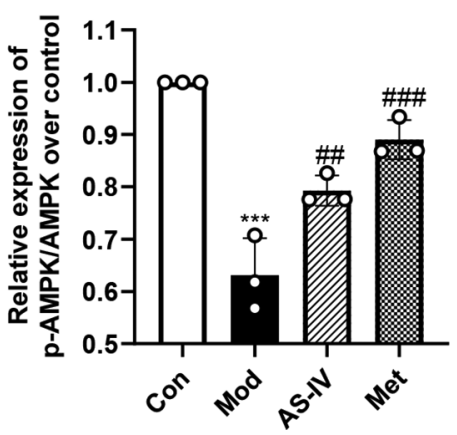

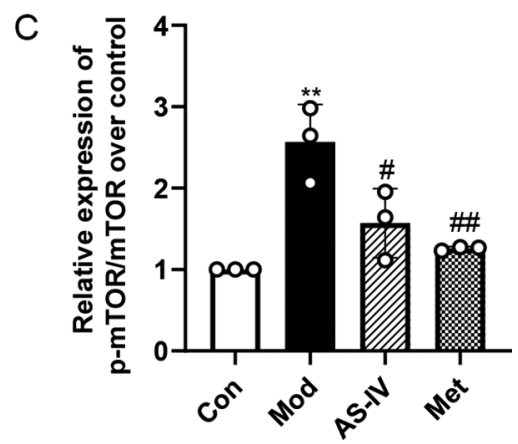

Figure 8. AS-IV activates autophagy via the AMPK/mTOR pathway in the liver of T2DM rats. (A) Protein expression levels of AMPK, p-AMPK, mTOR and $\mathrm{p}-\mathrm{mTOR}$ in liver tissues were detected via western blotting. (B and C) Semi-quantitative analysis of p-AMPK/AMPK ratio and p-mTOR/mTOR ratio. Data are presented as the mean $\pm \mathrm{SD}, \mathrm{n}=3 .{ }^{* *} \mathrm{P}<0.01,{ }^{* * * *} \mathrm{P}<0.001$ vs. control group; ${ }^{\#} \mathrm{P}<0.05,{ }^{\# \#} \mathrm{P}<0.01,{ }^{\# \# "} \mathrm{P}<0.001$ vs. model group. Con, Control; Mod, Model; AS-IV, Astragaloside IV ( $80 \mathrm{mg} / \mathrm{kg}$ ); Met, Metformin (200 mg/kg); p-, phosphorylated; AMPK, adenosine monophosphate-activated protein kinase.

to possess comprehensive pharmacological effects, especially in diabetes (32). Previous research has confirmed that AS-IV at a dose of $80 \mathrm{mg} / \mathrm{kg}$ is widely used in the study of liver diseases and other diseases in Sprague-Dawley rats $(33,34)$. Moreover, in our previous studies, a dose of $80 \mathrm{mg} / \mathrm{kg}$ AS-IV could significantly improve the complications of diabetic rats, such as diabetic nephropathy and cardiomyopathy $(18,19)$. Therefore, in the current study, a concentration of AS-IV $80 \mathrm{mg} / \mathrm{kg}$ was used to evaluate the protective effect on diabetic liver injury in T2DM.

In the present study, HFD combined with low-dose STZ was used to induce a diabetic liver injury model in T2DM rats $(18,19)$. It was found that the common manifestations of food intake, water intake and urine volume in T2DM were significantly increased, while body weight was significantly decreased in the T2DM model group. In addition, the levels of ALT, AST, FBG, FINS and GSP in serum and the liver index of liver weight to body weight were significantly increased. It was also observed that the pathological changes in the liver, including hepatocyte disorder, lipid deposition and collagen expression, were significantly increased in T2DM rats. Importantly, IR and dyslipidaemia appeared, and oxidative stress and inflammation levels were significantly increased in the liver. This confirmed the success of modelling in T2DM rats and the occurrence of diabetic liver injury. However, AS-IV treatment relieved the aforementioned symptoms, indicating that AS-IV exerted a protective effect on diabetic liver injury in T2DM rats. Additionally, the present findings revealed that AS-IV could promote liver autophagy in T2DM rats by modulating the AMPK/mTOR pathway.

Autophagy is a key self-repair mechanism and an important regulator of cellular homeostasis (7). The basal level of autophagy can adapt to various stresses and is essential for maintaining normal liver function (35). Accumulating evidence suggests that abnormal autophagy function is closely associated with diabetic liver injury $(36,37)$. The present study observed that the liver autophagy ability was impaired in T2DM rats. These results are consistent with previous studies, and provide further support for the role of autophagy in the occurrence of diabetic liver injury in T2DM $(36,37)$. The recovery or activation of autophagy has a protective effect on target organs $(10,38)$. Similarly, the present study found that AS-IV treatment normalised the autophagic activity in the liver of T2DM rats, suggesting that AS-IV activated the suppressed autophagy in the liver of T2DM rats. Furthermore, it has been reported that autophagy can ameliorate IR, dyslipidaemia, oxidative stress and inflammation (8-11). In summary, these findings indicate that autophagy can participate in the occurrence and development of diabetic liver injury in T2DM by improving IR, dyslipidaemia, oxidative stress and inflammation.

The relationship between autophagy, IR and dyslipidaemia is important in the treatment of diabetic liver injury in T2DM $(8,9,11)$. It has been reported that IR and dyslipidaemia are important risk factors of diabetic liver injury in T2DM (5). IR, a core feature of T2DM, is defined as the reduced efficiency of insulin in promoting glucose uptake and utilization, 
which is particularly critical for maintaining blood glucose stability (39). Moreover, IR can develop into obvious T2DM, accompanied by the loss of $\beta$-cell function, and eventually leading to the loss of $\beta$-cells (40). Dyslipidaemia usually exists in patients with T2DM, and becomes an important predictor of the development of T2DM (41). The accumulation of liver fat caused by dyslipidaemia leads to the weakening of the liver's detoxification ability (5). Additionally, the excessive accumulation of liver fat may aggravate IR and cause severe metabolic dysfunction $(5,6)$. Therefore, improving IR and dyslipidaemia has become an important method for the treatment of diabetic liver injury in T2DM.

Accumulating evidence has indicated that the enhancement of autophagy has been shown to improve IR and dyslipidaemia (36,42-44). Moreover, autophagy can maintain the function of pancreatic $\beta$-cells during the occurrence and development of diabetic liver injury in T2DM $(45,46)$. AS-IV has a protective effect on hepatic steatosis by attenuating IR and lipid deposition in HepG2 cells (47). In the present study, it was found that IR was significantly increased, and dyslipidaemia appeared in T2DM rats, while AS-IV treatment improved IR and dyslipidaemia. These current findings suggested that AS-IV treatment may improve the IR and dyslipidaemia in T2DM rats by enhancing liver autophagy.

The interaction of autophagy, oxidative stress and inflammation is crucial in the occurrence and development of diabetic liver injury in T2DM. Oxidative stress and inflammation are considered to be the main factors in the progression of diabetes complications $(6,48)$. Hyperglycaemia can induce acute oxidative stress, which stimulates tissue-specific inflammation and participates in the pathogenesis of T2DM $(48,49)$. Therefore, improving oxidative stress and inflammation has become an important strategy for treating diabetic liver injury in T2DM. It has been reported that autophagy improves cell damage by inhibiting oxidative stress and inflammation (50). Furthermore, AS-IV can protect the kidney from damage by inhibiting oxidative stress and inflammation in T2DM (51). The present study demonstrated that oxidative stress and inflammation levels were significantly increased in the liver of T2DM rats, and AS-IV treatment significantly improved oxidative stress and inflammation levels. These observations are consistent with other studies $(23,24)$. These data suggested that AS-IV treatment may attenuate oxidative stress and inflammation levels in the liver of T2DM rats by enhancing autophagy.

AMPK/mTOR-mediated autophagy has been reported to be involved in diabetes complications (52). Previous studies have revealed that AS-IV prevented podocyte injury by activating AMPK-mediated autophagy in diabetic nephropathy and promoted functional recovery after acute spinal cord injury by activating mTOR-mediated autophagy $(53,54)$. To identify the underlying mechanism of autophagy in diabetic liver injury in T2DM, the present study focused on the AMPK/mTOR pathway. It was found that the AMPK activity in the liver was decreased in T2DM rats, and was improved by AS-IV treatment. Furthermore, it was demonstrated that AS-IV suppressed the increase of mTOR activity in T2DM rats. The results suggested that AS-IV treatment may regulate liver autophagy via the AMPK/mTOR pathway in diabetic liver injury in T2DM rats.
There are several limitations to the present study. First, whether autophagy was caused by the activation of the AMPK/mTOR pathway requires further research. Second, the specific relationship between autophagy and IR, dyslipidaemia, oxidative stress and inflammation needs to be further investigated.

In conclusion, the present study demonstrated that AS-IV alleviated diabetic liver injury in T2DM rats, and its mechanism may be associated with the promotion of AMPK/mTOR-mediated autophagy, which improved IR, dyslipidaemia, oxidative stress and inflammation. The present results provide evidence for the protective effect of AS-IV in diabetic liver injury in T2DM. Furthermore, regulating autophagy may be an effective strategy to improve diabetic liver injury in T2DM.

\section{Acknowledgements}

The authors would like to thank Mrs. Zhirui Fang in the Department of Pharmacology, and Mr. Dake Huang in the Synthetic Laboratory of Basic Medicine College for their technical assistance.

\section{Funding}

This study was supported by grants from the National Natural Science Foundation of China (grant no. 81970630) and Major Science and Technology Projects in Anhui Province (grant no. 201903a07020025).

\section{Availability of data and materials}

The datasets used and/or analyzed during the current study are available from the corresponding authors on reasonable request.

\section{Authors' contributions}

YFZ performed the experiments, analyzed data and was a major contributor in writing the manuscript. YS and JZ assessed the authenticity of the raw data and ensured its legitimacy. YS and JZ helped with the statistical analysis. YHZ, YL, YH and XD helped to establish the animal model and revised the manuscript. WZL and WPL designed the study and critically revised the manuscript. All authors read and approved the final submitted manuscript.

\section{Ethics approval and consent to participate}

All animal experiments were conducted under protocols approved by the Ethics Review Committee of Anhui Medical University (approval no. LLSC20190302).

\section{Patient consent for publication}

Not applicable.

\section{Competing interests}

The authors declare that they have no competing interests. 


\section{References}

1. Tan SY, Mei Wong JL, Sim YJ, Wong SS, Mohamed Elhassan SA, Tan SH, Ling Lim GP, Rong Tay NW, Annan NC, Bhattamisra SK and Candasamy M: Type 1 and 2 diabetes mellitus: A review on current treatment approach and gene therapy as potential intervention. Diabetes Metab Syndr 13: 364-372, 2019.

2. Zheng Y, Ley SH and Hu FB: Global aetiology and epidemiology of type 2 diabetes mellitus and its complications. Nat Rev Endocrinol 14: 88-98, 2018

3. Shima T,Uto H,Ueki K, Takamura T, Kohgo Y, Kawata S, Yasui K, Park H, Nakamura N, Nakatou T, et al: Clinicopathological features of liver injury in patients with type 2 diabetes mellitus and comparative study of histologically proven nonalcoholic fatty liver diseases with or without type 2 diabetes mellitus J Gastroenterol 48: 515-525, 2013.

4. Hsiang JC, Gane EJ, Bai WW and Gerred SJ: Type 2 diabetes: A risk factor for liver mortality and complications in hepatitis B cirrhosis patients. J Gastroenterol Hepatol 30: 591-599, 2015.

5. Bedi O, Aggarwal S, Trehanpati N, Ramakrishna G and Krishan P: Molecular and pathological events involved in the pathogenesis of diabetes-associated nonalcoholic fatty liver disease. J Clin Exp Hepatol 9: 607-618, 2019.

6. Akash MS, Rehman K and Chen S: Role of inflammatory mechanisms in pathogenesis of type 2 diabetes mellitus. J Cell Biochem 114: 525-531, 2013

7. Mizushima N and Komatsu M: Autophagy: Renovation of cells and tissues. Cell 147: 728-741, 2011

8. Liu H, Javaheri A, Godar RJ, Murphy J, Ma X, Rohatgi N, Mahadevan J, Hyrc K, Saftig P, Marshall C, et al: Intermittent fasting preserves beta-cell mass in obesity-induced diabetes via the autophagy-lysosome pathway. Autophagy 13: 1952-1968, 2017.

9. Chatterjee T, Pattanayak R, Ukil A, Chowdhury S and Bhattacharyya M: Autophagy protects peripheral blood mononuclear cells against inflammation, oxidative and nitrosative stress in diabetic dyslipidemia. Free Radic Biol Med 143: 309-323, 2019.

10. Fan W, Han D, Sun Z, Ma S, Gao L, Chen J, Li X, Li X, Fan M, $\mathrm{Li} \mathrm{C}$, et al: Endothelial deletion of mTORC1 protects against hindlimb ischemia in diabetic mice via activation of autophagy, attenuation of oxidative stress and alleviation of inflammation. Free Radic Biol Med 108: 725-740, 2017.

11. Martinez-Lopez N and Singh R: Autophagy and lipid droplets in the liver. Annu Rev Nutr 35: 215-237, 2015.

12. Zhang BB, Zhou G and Li C: AMPK: An emerging drug target for diabetes and the metabolic syndrome. Cell Metab 9: 407-416, 2009.

13. Kim J, Kundu M, Viollet B and Guan KL: AMPK and mTOR regulate autophagy through direct phosphorylation of Ulk1. Nat Cell Biol 13: 132-141, 2011.

14. Liu TY, Xiong XQ, Ren XS, Zhao MX, Shi CX, Wang JJ, Zhou YB, Zhang F, Han Y, Gao XY, et al: FNDC5 alleviates hepatosteatosis by restoring AMPK/mTOR-mediated autophagy, fatty acid oxidation, and lipogenesis in mice. Diabetes 65 3262-3275, 2016

15. Yang Y, Gao J, Zhang Y, Xu W, Hao Y, Xu Z and Tao L: Natural pyrethrins induce autophagy of HepG2 cells through the activation of AMPK/mTOR pathway. Environ Pollut 241: 1091-1097, 2018.

16. Wang E, Wang L, Ding R, Zhai M, Ge R, Zhou P, Wang T, Fang $\mathrm{H}$, Wang $\mathrm{J}$ and Huang J: Astragaloside IV acts through multi-scale mechanisms to effectively reduce diabetic nephropathy. Pharmacol Res 157: 104831, 2020

17. Song MT, Ruan J, Zhang RY, Deng J, Ma ZQ and Ma SP: Astragaloside IV ameliorates neuroinflammation-induced depressive-like behaviors in mice via the PPAR $\gamma / \mathrm{NF}-\kappa \mathrm{B} / \mathrm{NLRP} 3$ inflammasome axis. Acta Pharmacol Sin 39: 1559-1570, 2018.

18. Ju Y, Su Y, Chen Q, Ma K, Ji T, Wang Z, Li W and Li W: Protective effects of Astragaloside IV on endoplasmic reticulum stress-induced renal tubular epithelial cells apoptosis in type 2 diabetic nephropathy rats. Biomed Pharmacother 109: 84-92,2019.

19. Wang Z, Zhu Y,Zhang Y, Zhang J, Ji T, Li W and Li W: Protective effects of AS-IV on diabetic cardiomyopathy by improving myocardial lipid metabolism in rat models of T2DM. Biomed Pharmacother 127: 110081, 2020.

20. Antuna-Puente B, Disse E, Rabasa-Lhoret R, Laville M, Capeau J and Bastard JP: How can we measure insulin sensitivity/resistance? Diabetes Metab 37: 179-188, 2011.
21. Xu L, Yu Y, Sang R, Li J, Ge B and Zhang X: Protective effects of taraxasterol against ethanol-induced liver injury by regulating CYP2E1/Nrf2/HO-1 and NF- $\mathrm{BB}$ signaling pathways in mice. Oxid Med Cell Longev 2018: 8284107, 2018.

22. Michalopoulos GK: Hepatostat: Liver regeneration and normal liver tissue maintenance. Hepatology 65: 1384-1392, 2017.

23. Bao L, Li J, Zha D, Zhang L, Gao P, Yao T and Wu X: Chlorogenic acid prevents diabetic nephropathy by inhibiting oxidative stress and inflammation through modulation of the Nrf2/HO-1 and NF- $\kappa$ B pathways. Int Immunopharmacol 54: 245-253, 2018.

24. Guo M, Lu H, Qin J, Qu S, Wang W, Guo Y, Liao W, Song M, Chen J and Wang Y: Biochanin A provides neuroprotection against cerebral Ischemia/Reperfusion Injury by Nrf2-mediated inhibition of oxidative stress and inflammation signaling pathway in rats. Med Sci Monit 25: 8975-8983, 2019.

25. Ding GB, Sun J, Wu G, Li B, Yang P, Li Z and Nie G: Robust anticancer efficacy of a biologically synthesized tumor acidity-responsive and Autophagy-Inducing functional beclin 1. ACS Appl Mater Interfaces 10: 5227-5239, 2018.

26. Hanada T, Noda NN, Satomi Y, Ichimura Y, Fujioka Y, Takao T, Inagaki $\mathrm{F}$ and Ohsumi Y: The Atg12-Atg5 conjugate has a novel E3-like activity for protein lipidation in autophagy. J Biol Chem 282: 37298-37302, 2007.

27. Bardag-Gorce F, Francis T, Nan L, Li J, He Lue Y, French BA and French SW: Modifications in P62 occur due to proteasome inhibition in alcoholic liver disease. Life Sci 77: 2594-2602, 2005.

28. Rao Z, Pan X, Zhang H, Sun J, Li J, Lu T, Gao M, Liu S, Yu D and Ding Z: Isoflurane preconditioning alleviated murine liver ischemia and reperfusion injury by restoring AMPK/mTOR-mediated autophagy. Anesth Analg 125: 1355-1363, 2017.

29. Han D: Treatment with astragaloside IV reduced blood glucose, regulated blood lipids, and protected liver function in diabetic rats. J Int Med Res 49: 300060519841165, 2021.

30. Zhang Y, Cao Y, Chen J, Qin H and Yang L: A New possible mechanism by which punicalagin protects against liver injury induced by type 2 diabetes mellitus: Upregulation of autophagy via the Akt/FoxO3a signaling pathway. J Agric Food Chem 67: 13948-13959, 2019

31. Liang W, Zhang D, Kang J, Meng X, Yang J, Yang L, Xue N, Gao Q, Han S and Gou X: Protective effects of rutin on liver injury in type 2 diabetic $\mathrm{db} / \mathrm{db}$ mice. Biomed Pharmacother 107: 721-728, 2018.

32. Gao K, Yang R, Zhang J, Wang Z, Jia C, Zhang F, Li S, Wang J, Murtaza G, Xie H, et al: Effects of Qijian mixture on type 2 diabetes assessed by metabonomics, gut microbiota and network pharmacology. Pharmacol Res 130: 93-109, 2018.

33. Qu X, Gao H, Tao L, Zhang Y, Zhai J, Sun J, Song Y and Zhang S: Astragaloside IV protects against cisplatin-induced liver and kidney injury via autophagy-mediated inhibition of NLRP3 in rats. J Toxicol Sci 44: 167-175, 2019.

34. Liu W, Liu G and Liu J: Effects of astragaloside IV on the pharmacokinetics of omeprazole in rats. Pharm Biol 57: 449-452, 2019.

35. Zhang Q, Li Y, Liang T, Lu X, Zhang C, Liu X, Jiang X, Martin RC, Cheng M and Cai L: ER stress and autophagy dysfunction contribute to fatty liver in diabetic mice. Int J Biol Sci 11: 559-568, 2015

36. Lin CW, Zhang H, Li M, Xiong X, Chen X, Chen X, Dong XC and Yin XM: Pharmacological promotion of autophagy alleviates steatosis and injury in alcoholic and non-alcoholic fatty liver conditions in mice. J Hepatol 58: 993-999, 2013.

37. Zhang F, Zhao S, Yan W, Xia Y, Chen X, Wang W, Zhang J, Gao C, Peng C, Yan F, et al: Branched chain amino acids cause liver injury in obese/diabetic mice by promoting adipocyte lipolysis and inhibiting hepatic autophagy. EBioMedicine 13: 157-167, 2016.

38. Yao Q, Ke ZQ, Guo S, Yang XS, Zhang FX, Liu XF, Chen X, Chen HG, Ke HY and Liu C: Curcumin protects against diabetic cardiomyopathy by promoting autophagy and alleviating apoptosis. J Mol Cell Cardiol 124: 26-34, 2018.

39. Artunc F, Schleicher E, Weigert C, Fritsche A, Stefan N and Häring HU: The impact of insulin resistance on the kidney and vasculature. Nat Revi Nephrol 12: 721-737, 2016.

40. Jourdan T, Godlewski G and Kunos G: Endocannabinoid regulation of $\beta$-cell functions: Implications for glycaemic control and diabetes. Diabetes Obes Metab 18: 549-557, 2016.

41. Ahmadieh $\mathrm{H}$ and Azar ST: Liver disease and diabetes: Association, pathophysiology, and management. Diabetes Res Clin Pract 104: 53-62, 2014 
42. Liu HY, Han J, Cao SY, Hong T, Zhuo D, Shi J, Liu Z and Cao W: Hepatic autophagy is suppressed in the presence of insulin resistance and hyperinsulinemia: Inhibition of FoxO1-dependent expression of key autophagy genes by insulin. J Biol Chem 284: 31484-31492, 2009.

43. Codogno P and Meijer AJ: Autophagy: A potential link between obesity and insulin resistance. Cell Metabolism 11: 449-451, 2010.

44. Li R, Guo E, Yang J, Li A, Yang Y, Liu S, Liu A and Jiang X: $1,25(\mathrm{OH})_{2} \mathrm{D}_{3}$ attenuates hepatic steatosis by inducing autophagy in mice. Obesity (Silver Spring) 25: 561-571, 2017.

45. Jung HS, Chung KW, Won Kim J, Kim J, Komatsu M, Tanaka K, Nguyen YH, Kang TM, Yoon KH, Kim JW, et al: Loss of autophagy diminishes pancreatic beta cell mass and function with resultant hyperglycemia. Cell Metab 8: 318-324, 2008.

46. Mir SU, George NM, Zahoor L, Harms R, Guinn Z and Sarvetnick NE: Inhibition of autophagic turnover in $\beta$-cells by fatty acids and glucose leads to apoptotic cell death. J Biol Chem 290: 6071-6085, 2015

47. Wang $\mathrm{C}, \mathrm{Li} \mathrm{Y}, \mathrm{Hao} \mathrm{M}$ and $\mathrm{Li} \mathrm{W}$ : Astragaloside IV inhibits triglyceride accumulation in insulin-resistant HepG2 cells via AMPK-induced SREBP-1c phosphorylation. Front Pharmacol 9: 345, 2018.

48. Wu MY, Yiang GT and Lai TT: The Oxidative stress and mitochondrial dysfunction during the pathogenesis of diabetic retinopathy. Oxid Med Cell Longev 2018: 3420187, 2018.

49. Zhang Y, Yuan D, Yao W, Zhu Q, Liu Y, Huang F, Feng J, Chen X, Huang Y, Chi X and Hei Z: Hyperglycemia aggravates hepatic ischemia reperfusion injury by inducing chronic oxidative stress and inflammation. Oxid Med Cell Longev 2016: 3919627, 2016.
50. Meng L, Zhao X and Zhang H: HIPK1 interference attenuates inflammation and oxidative stress of acute lung injury via autophagy. Med Sci Monit 25: 827-835, 2019.

51. He KQ, Li WZ, Chai XQ, Yin YY, Jiang Y and Li WP Astragaloside IV prevents kidney injury caused by iatrogenic hyperinsulinemia in a streptozotocin-induced diabetic rat model. Int J Mol Med 41: 1078-1088, 2018.

52. Yang F, Zhang L, Gao Z, Sun X, Yu M, Dong S, Wu J, Zhao Y, $\mathrm{Xu} \mathrm{C}$, Zhang $\mathrm{W}$ and Lu F: Exogenous $\mathrm{H} 2 \mathrm{~S}$ protects against diabetic cardiomyopathy by activating autophagy via the AMPK/mTOR pathway. Cell Physiol Biochem 43: 1168-1187, 2017.

53. Guo H, Wang Y, Zhang X, Zang Y, Zhang Y, Wang L, Wang H, Wang Y, Cao A and Peng W: Astragaloside IV protects against podocyte injury via SERCA2-dependent ER stress reduction and AMPK $\alpha$-regulated autophagy induction in streptozotocin-induced diabetic nephropathy. Sci Rep 7: 6852, 2017.

54. Lin J, Pan X, Huang C, Gu M, Chen X, Zheng X, Shao Z, Hu S, Wang B, Lin H, et al: Dual regulation of microglia and neurons by Astragaloside IV-mediated mTORC1 suppression promotes functional recovery after acute spinal cord injury. J Cell Mol Med 24: 671-685, 2020.

This work is licensed under a Creative Commons Attribution-NonCommercial-NoDerivatives 4.0 International (CC BY-NC-ND 4.0) License. 